\title{
Neocortical Activation of the Hippocampus during Sleep in Infant Rats
}

\author{
Ethan J. Mohns and Mark S. Blumberg \\ Department of Psychology and Delta Center, University of Iowa, Iowa City, Iowa 52242
}

We recently reported that the majority of hippocampal neurons in newborn rats increase their activity in association with myoclonic twitches, which are indicative of active sleep. Because spindle bursts in the developing somatosensory neocortex occur in response to sensory feedback from myoclonic twitching, we hypothesized that the state-dependent activity of the newborn hippocampus arises from sensory feedback that sequentially activates the neocortex and then hippocampus, constituting an early form of neocortical-hippocampal communication. Here, in unanesthetized 5- to 6-d-old rats, we test this hypothesis by recording simultaneously from forelimb and barrel regions of somatosensory neocortex and dorsal hippocampus during periods of spontaneous sleep and wakefulness and in response to peripheral stimulation. Myoclonic twitches were consistently followed by neocortical spindle bursts, which were in turn consistently followed by bursts of hippocampal unit activity; moreover, spindle burst power was positively correlated with hippocampal unit activity. In addition, exogenous stimulation consistently evoked this neocortical-to-hippocampal sequence of activation. Finally, parahippocampal lesions that disrupted functional connections between the neocortex and hippocampus effectively disrupted the transmission of both spontaneous and evoked neocortical activity to the hippocampus. These findings suggest that sleep-related motor activity contributes to the development of neocortical and hippocampal circuits and provides a foundation on which coordinated activity between these two forebrain structures develops.

\section{Introduction}

The neocortex and hippocampus of adult mammals cooperate with one another to enable the performance of complex cognitive tasks (Squire, 1992; Zola-Morgan and Squire, 1993; Aggleton et al., 2000; Lavenex and Amaral, 2000; Rolls, 2000). This cooperation requires efficient bidirectional interactions through which specific information is communicated via precise sequences of neuronal firing and collectively generated rhythms (Siapas and Wilson, 1998; Sirota et al., 2003, 2008; Jones and Wilson, 2005; Siapas et al., 2005; Ji and Wilson, 2007). It has been demonstrated in both anesthetized and sleeping rodents that the membrane potentials of hippocampal neurons can be modulated by neocortical "up states" and "down states" (Hahn et al., 2006, 2007; Isomura et al., 2006); moreover, this modulation appears to reach the hippocampus via the entorhinal cortex (Isomura et al., 2006).

In neonatal rats, the neocortex and hippocampus exhibit brief bursts of activity that punctuate an otherwise sparse EEG (Leinekugel et al., 2002; Khazipov et al., 2004). These electrographic patterns observed early in ontogeny are thought to facilitate activity-dependent development and refinement of cortical

Received Sept. 26, 2009; revised Jan. 17, 2010; accepted Jan. 20, 2010.

This work was supported by National Institute of Mental Health Research Grant MH50701 and Independent Scientist Award MH66424 (M.S.B.). We thank Cynthia Shaw, Amy Jo Marcano-Reik, and James Gibson for technical assistance, and Janet Best for statistical advice.

Correspondence should be addressed to Dr. Mark S. Blumberg, Department of Psychology, University of lowa, lowa City, IA 52242. E-mail: mark-blumberg@uiowa.edu.

E. J. Mohns's present address: Howard Hughes Medical Institute, Janelia Farm Research Campus, Ashburn, VA 20147.

DOI:10.1523/JNEUROSCI.4832-09.2010

Copyright $\odot 2010$ the authors $\quad 0270-6474 / 10 / 303438-12 \$ 15.00 / 0$ circuits (Katz and Shatz, 1996; Feller, 1999; O'Donovan, 1999). Indeed, within both the neocortex and hippocampus, morphology, excitability, and connectivity are shaped by activity (Corner et al., 2002; Lauri et al., 2003; Kirov et al., 2004; Le Bé and Markram, 2006).

We recently reported that the majority of hippocampal neurons in newborn rats increase their activity during periods of active sleep (AS), a behavioral state characterized by myoclonic twitching of skeletal muscles throughout the body (Mohns and Blumberg, 2008). A subset of these neurons displayed distinct bursts of activity immediately after limb twitches, suggesting that the twitches may provide sensory feedback to the developing hippocampus. Importantly, twitches have previously been shown to provide sensory feedback to the developing neocortex, where they produce brief "spindle burst" oscillations (Khazipov et al., 2004). Based on these findings, we hypothesized that twitchrelated neocortical activity subsequently activates the hippocampus by way of the entorhinal cortex.

This hypothesis was tested here by recording neocortical and hippocampal activity simultaneously in head-immobilized unanesthetized rats on postnatal day 5 (P5) to P6. Our results demonstrate that neocortical spindle burst oscillations consistently precede bursts of hippocampal unit activity and that greater spindle burst power is positively correlated with greater hippocampal unit activity. This neocortical-to-hippocampal sequence of activation was exhibited spontaneously in relation to myoclonic twitching and was also evoked by peripheral sensory stimulation. Parahippocampal lesions that disconnected the neocortex and hippocampus disrupted this sequential relationship, thus demonstrating for the first time that the activity of the neocortex 
causally influences activity in the hippocampus during early postnatal development.

\section{Materials and Methods}

All experiments were performed in accordance with the National Institutes of Health Guide for the Care and Use of Laboratory Animals and were approved by the Institutional Animal Care and Use Committee of the University of Iowa.

\section{Subjects}

Sixteen P5-P6 Sprague Dawley Norway rats (Rattus norvegicus) from 14 litters were used (12 P5, 4 P6; 12 males, 4 females; body weight, 10.9-17.5 g). Litters were culled to eight pups on the third day after birth (day of birth, day 0 ). Mothers and their litters were housed in standard laboratory cages $(48 \times 20 \times 26 \mathrm{~cm})$ in the animal colony at the University of Iowa where food and water were available ad libitum. All subjects were maintained on a $12 \mathrm{~h}$ light/dark schedule with lights on at 7:00 A.M., and all tests were conducted between 12:00 P.M. and 5:00 P.M.

\section{Surgery}

Under isoflurane anesthesia, the subject's skull was exposed, and then bleached, dried, and coated with Vetbond ( $3 \mathrm{M})$ to add strength. All holes necessary for probe implantation were drilled under anesthesia. For the hippocampus, coordinates were $2 \mathrm{~mm}$ posterior to bregma and 1-2 $\mathrm{mm}$ lateral from midline; for the $\mathrm{S} 1$-forelimb region, coordinates were $1 \mathrm{~mm}$ anterior to bregma and $3 \mathrm{~mm}$ lateral from midline; for the S1-barrel region, coordinates were $2 \mathrm{~mm}$ posterior to bregma and $3 \mathrm{~mm}$ lateral from midline. To secure the subject's head during testing, a custom-built stainless-steel apparatus (Karlsson et al., 2005), designed to attach to the ear bar and nose bar holders of a stereotaxic apparatus (David Kopf Instruments), was attached to the outer edges of the pretreated skull using cyanoacrylate adhesive gel. EMG electrodes (50 $\mu$ m diameter; California Fine Wire) were implanted into the forelimb, hindlimb, and nuchal muscles contralateral to the neural recording sites to identify behavioral state (Karlsson and Blumberg, 2002). Finally, to inhibit movement and calm the subject, it was wrapped gently in gauze (Corner and Kwee, 1976; Karlsson et al., 2005). The subject recovered from surgery for at least $1.5 \mathrm{~h}$ in a humidified incubator maintained at thermoneutrality $\left(35^{\circ} \mathrm{C}\right)$.

After recovery from surgery, the subject was transferred to a stereotaxic apparatus. The skull was leveled in the horizontal plane, and recording electrodes were lowered into the predrilled holes. All subjects were given a $2 \mathrm{~h}$ acclimation period to adjust to the stereotaxic apparatus, to achieve physiological body and brain temperatures, and to exhibit distinct periods of high and low nuchal muscle tone. During all experiments, body and brain temperatures were maintained at $\sim 35$ and $37^{\circ} \mathrm{C}$, respectively. If at any point during the procedure the subject appeared to be in distress, the experiment was terminated. After 10-15 min baseline recordings had been made, exogenous stimuli were applied to the forelimb and whisker pad and the responses within the neocortex and hippocampus were analyzed. Electrical stimuli were delivered to the forelimb contralateral to the neural recording sites $(\sim 10 \mathrm{~V} ; 100 \mu \mathrm{s} ; \geq 20$ stimuli); these stimuli were generated using a stimulator (Grass Technologies) and were delivered via surgically implanted wires (50 $\mu \mathrm{m}$ diameter; California Fine Wire). Electrical stimulation of the forelimb was followed by $\geq 20$ whisker pad stimulations, applied using single rostralto-caudal strokes with a fine paintbrush (also contralateral to neural recording sites). Stimuli were applied intermittently during wakefulness, quiet sleep (QS), and AS, and the time of delivery for each stimulus was marked by simultaneously pressing a key on a keyboard.

\section{Recording procedure and data acquisition}

Hippocampal recordings were performed using 16-site silicon probes (four recording sites on each of four parallel shanks; $100 \mu \mathrm{m}$ vertical separation between recording sites; NeuroNexus Technologies) lowered into the dorsal CA1. An insulated silver wire (Medwire; $0.25 \mathrm{~mm}$ diameter) inserted into the cerebellum served as a reference electrode. Silicon probes were connected to a unity-gain headstage and digital amplifier (Tucker-Davis Technologies) that amplified $(10,000 \times)$ and filtered (1-
$3000 \mathrm{~Hz}$ bandpass) the neural signals. Neocortical EEG recordings were performed using custom-made chlorinated silver $(\mathrm{Ag} / \mathrm{AgCl})$ electrodes (Medwire; $0.25 \mathrm{~mm}$ diameter); signals from the latter were amplified using an A-M Systems amplifier. A $60 \mathrm{~Hz}$ notch filter was applied during all recording sessions. EMG signals were amplified $(10,000 \times)$ and filtered (300-5000 Hz bandpass) using a differential amplifier (A-M Systems). Neural and EMG signals were sampled at $12.5 \mathrm{kHz}$ using a digital interface (Cambridge Electronic Design) and recorded synchronously to hard disk for off-line analysis.

\section{Data analysis}

Identification of behavioral state. For each subject, EMG signals were dichotomized into periods of high tone (indicative of wakefulness) and hypotonia/atonia (indicative of sleep) using methods described previously (Karlsson et al., 2005). Sleep bouts were further divided into periods of QS and AS. Whereas QS is characterized by hypotonia/atonia and behavioral quiescence, AS is characterized by the appearance of myoclonic twitches against a background of muscle atonia (Jouvet-Mounier et al., 1970; Karlsson et al., 2005; Seelke and Blumberg, 2008). To assess the relationship between AS and hippocampal activity, we identified each individual twitch in each record. To do this, we used the off-line method for detecting twitches and converting them from continuous EMG data into digitally represented events described by Mohns and Blumberg (2008).

Identification of hippocampal unit activity. In CA1, four recording channels containing heterogeneous unit activity were selected for analysis. Using Spike2 software (Cambridge Electronic Design), each of the selected channels was filtered $(300-3000 \mathrm{~Hz}$ bandpass $)$ and a digital threshold was then set to extract unit data with a signal-to-noise ratio of at least 2:1. Principal components analysis was then used for spike sorting. The sorted units were assigned to groups using graphical cluster cutting. A cluster was judged to contain a single unit only if the autocorrelation analysis indicated a refractory period of at least $2 \mathrm{~ms}$.

Construction of twitch cross-correlograms. The temporal correlations among twitches occurring in multiple muscle groups were analyzed using twitch cross-correlograms. To construct twitch cross-correlograms, all twitches were first converted from continuous EMG data into digitally represented events (Mohns and Blumberg, 2008). Because twitches were then represented in the same format and on the same timescale as action potentials, cross-correlograms could be computed using methods similar to those used to compute cross-correlograms for unit data (Eggermont, 1992). The number of coincident twitches in muscle groups A and B was determined within $\pm 100 \mathrm{~ms}$ (in $10 \mathrm{~ms}$ bins) of the trigger twitch. The expected value $(E)$ of the cross-correlogram, under the assumption of independence of the groups, is equal to the product of the number of twitches in groups $\mathrm{A}$ and $\mathrm{B}$, divided by the number of bins in the record. The value of $E$ is thereby normalized by twitch count. The $\mathrm{SD}$ of $E$ is then equal to $E^{1 / 2}$, and we considered the correlation between twitches in any two muscle groups to be significant when at least 1 of the 20 bins in the crosscorrelogram had a value $\geq E+3$ SDs ( $p<0.0015$, two-tailed). Here, and in subsequent analyses, this conservative $\alpha$ was chosen to help rectify issues related to multiple comparisons and the nonrandom distribution of the signals being examined.

Quantification of the relationship between myoclonic twitches and neocortical spindle bursts. Spindle bursts were identified using methods identical with those used by Marcano-Reik and Blumberg (2008), and were prepared for analysis by bandpass filtering $(5-40 \mathrm{~Hz})$ the local field potential (LFP) traces, and then calculating the root mean square of the filtered data in $10 \mathrm{~ms}$ bins. This provided a continuous measure of spindle burst power that could be related to twitches. For each subject, spindle burst power during AS-related twitching was quantified by calculating the twitchtriggered $( \pm 500 \mathrm{~ms})$ mean power for the prepared traces. We considered spindle burst power to be significantly associated with twitching when mean twitch-triggered power was $\geq 3$ SDs $(p<0.0015)$ above the randomtriggered (baseline) mean power.

Analysis of twitch-related hippocampal unit activity. To quantitatively determine the manner in which each unit fired during periods of twitching, we constructed twitch-triggered event correlations in which unit activity was summed for the period within $\pm 500 \mathrm{~ms}$ (in $10 \mathrm{~ms}$ bins) of a 
A
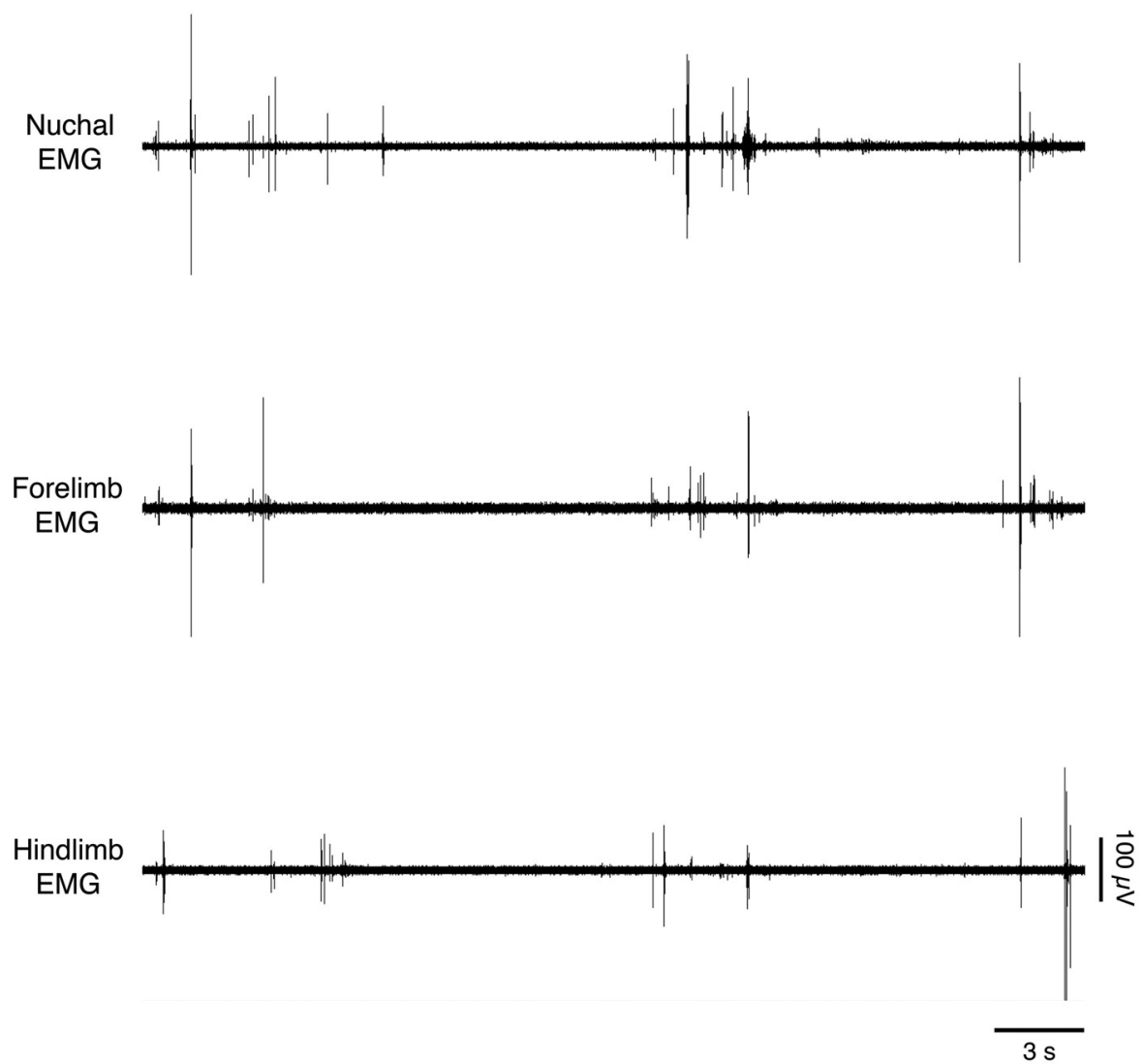

$\mathrm{B}$

Nuchal-Forelimb Twitch Correlation

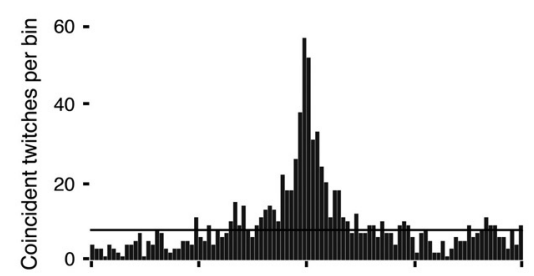

Hindlimb-Forelimb Twitch Correlation

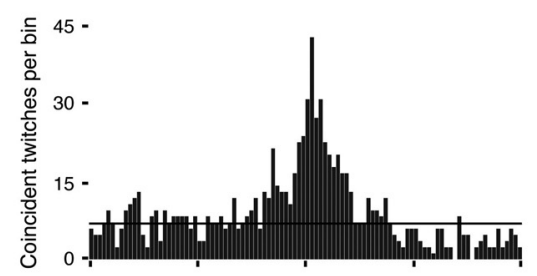

Hindlimb-Nuchal Twitch Correlation

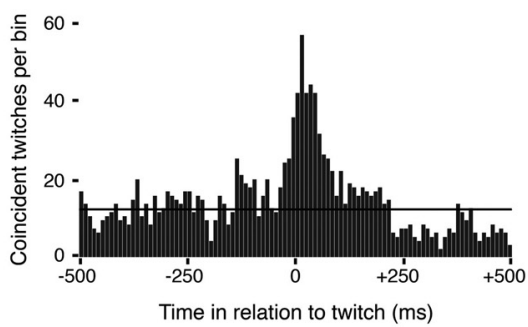

Figure 1. Correlated bouts of myoclonic twitches in multiple muscle groups. $A$, Representative data from a P5 rat showing nuchal, forelimb, and hindlimb EMG activity during a period of sleep. Bursts of activity visible in the EMG records indicate myoclonic twitching. Note that individual twitches in separate muscle groups tend to occur in close proximity to one another. $\boldsymbol{B}$, Representative cross-correlograms from a single subject illustrate the tendency for twitches to be correlated between pairs of muscle groups. The horizontal line in each cross-correlogram indicates the expected value +3 SDs $(p<0.0015)$.

twitch. This analysis was similar to the twitch-triggered peristimulus unit histograms used by Mohns and Blumberg (2008) but allowed for more accurate sampling of unit activity within bouts of twitching. The twitchtriggered event correlation of each unit was compared with a second event correlation that was triggered by randomized twitches. The random-triggered event correlation provided a baseline mean and SD to use for determining whether the twitch-triggered activity was significant; we considered an event correlation to be significant when its twitchtriggered mean was $\geq 3$ SDs above its baseline mean ( $p<0.0015$ ). For each unit, the number of significant unit bins (i.e., those reaching or exceeding criterion) occurring before and after the trigger twitch was determined, and the number of significant pre-twitch bins was plotted against the number of significant post-twitch bins.

Quantification of the relationship between neocortical spindle bursts and hippocampal unit activity. The relationship between spindle bursts and hippocampal unit activity was analyzed by first obtaining, for each unit, the mean unit-triggered spindle burst power $( \pm 500 \mathrm{~ms} ; 10 \mathrm{~ms}$ bins $)$ in the S1-forelimb and S1-barrel regions. We next compared these averages to a second set of averages generated by randomizing the trigger unit. We considered there to be a significant association between neocortical spindle burst power and hippocampal unit activity when the average unittriggered spindle burst power was $\geq 3$ SDs above the random-triggered (baseline) mean $(p<0.0015)$.

Response to exogenous sensory stimulation. In each subject, a stimulus was considered to evoke significant neocortical spindle burst power when the mean stimulus-triggered power $( \pm 500 \mathrm{~ms} ; 10 \mathrm{~ms}$ bins $)$ was greater than or equal to the subject's previously established baseline mean +3 SDs $(p<0.0015)$. To determine whether hippocampal units responded significantly to a stimulus, the previously marked times of stimulus delivery were randomized, and a random-triggered event correlation ( $+1 \mathrm{~s} ; 10 \mathrm{~ms}$ bins) was computed, providing a baseline mean and SD. Units with five or more significant bins (i.e., those exceeding the baseline mean +3 SDs; $p<0.0015)$ during the 1 s poststimulus period were considered significantly responsive to the stimulus.

Electrical stimulation was delivered to the forelimb in six of the seven subjects; the excluded subject had a nonfunctional stimulation electrode. In each of these six subjects, stimulation was followed by a significant $(p<0.0015)$ increase in spindle burst power in the $\mathrm{S} 1$-forelimb region of the neocortex; however, we also found a significant $(p<0.0015)$ increase in spindle burst power in the S1-barrel region of the four subjects that had functional S1-barrel electrodes. In our experiments, the electrical stimulus was thus not capable of producing discrete activation of the S1-forelimb region; rather, the stimulus tended to activate both regions of S1 simultaneously. Importantly, the EMG records indicated that this diffuse activation was not attributable to arousal from sleep. Thus, because it is uncertain whether such electrical impulses provide discrete sensory stimulation, the results of these stimulations were not considered further. These findings differ from those of Khazipov et al. (2004), in which somatotopic activation of S1 was observed after electrical stimulation; however, it is possible that this difference is accounted for by differences in experimental methodology.

Next, manual whisker pad stimulation was delivered to five of the seven subjects. In four of these subjects, stimulation was followed by a significant $(p<0.0015)$ increase in spindle burst power in the S1-barrel region; the remaining subject displayed an increase in spindle burst power that was significant at $p<0.010$ (2.5 SDs). As with electrical 

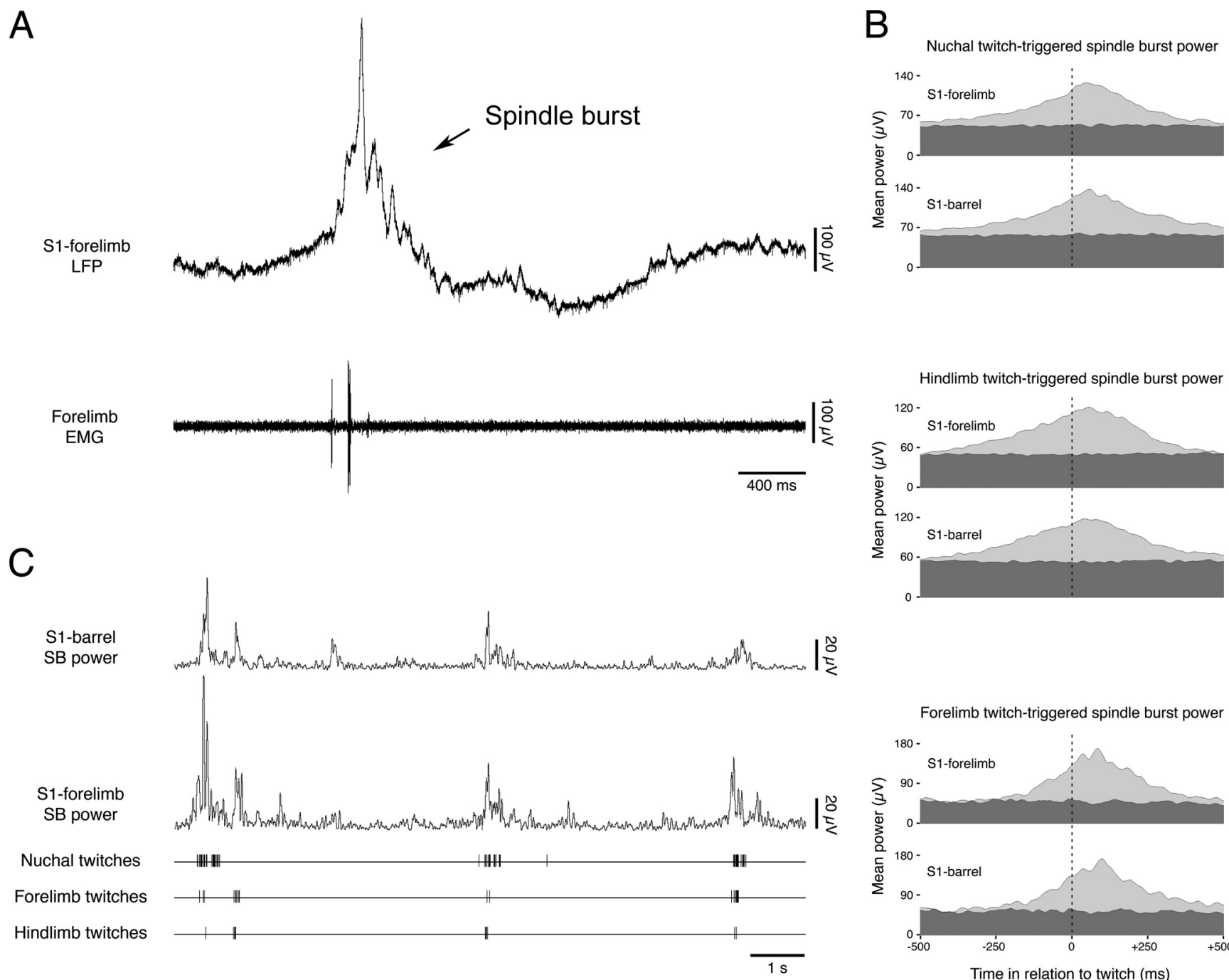

Figure 2. Correlated muscle twitches precede correlated spindle bursts in S1. A, Representative LFP and EMG recordings from the forelimb region of somatosensory (S1) neocortex and forelimb muscle, respectively. Note the temporal relation between the spindle burst and muscle twitch. B, Analyses of twitch-triggered spindle burst power (5-40Hz) in the S1-forelimb and S1-barrel regions. The vertical dotted lines at $0 \mathrm{~ms}$ indicate the time of twitch occurrence. Twitch-triggered spindle burst power is shown in light gray; baseline (random-triggered) spindle burst power is shown in dark gray. Note that a marked increase in spindle burst power consistently follows twitch occurrence in both areas of neocortex. C, Representative recording depicting spindle burst power in the S1-forelimb and S1-barrel regions in relation to digitized nuchal, forelimb, and hindlimb muscle twitches. Note that the increases in spindle burst power are associated with correlated bouts of twitching.

stimulations, manual whisker pad stimulations also caused significant increases in spindle burst power in the S1-forelimb regions of these subjects. However, follow-up experiments revealed that, consistent with the findings of Khazipov et al. (2004), manual stimulation of the whiskers themselves was capable of eliciting S1-barrel-specific responses; likewise, manual stimulation of the forelimb was capable of eliciting S1-forelimbspecific responses. Thus, whereas electrical stimulation appeared to result in global activation of the developing neocortex, manual stimulation appeared to be capable of eliciting region-specific responses, suggesting that the latter is interpreted by the developing neocortex as a typical somatosensory stimulus.

Nonetheless, in the present experiments, the manual stimulation was applied to the entire whisker pad, including both the whiskers and the underlying facial skin, including broader regions of the side of the subject's face. Follow-up experiments revealed that, unlike the more focal activation of the S1-barrel region after stimulation of the whiskers alone, the less precise whisker pad stimulation used in these experiments consistently resulted in additional activation of neighboring neocortical areas, including the S1-forelimb region. Because of its lack of neocortical specificity, the whisker pad stimulation is viewed here as a general sensory evoked potential, rather than as a topographically specific sensory stimulus.

\section{Parahippocampal lesions}

Surgical disconnection of the neocortex from the hippocampus was accomplished by transecting the parahippocampal region, which is the primary route of information transfer between the two structures. Transections were performed under isoflurane anesthesia, using a 25 gauge needle to manually drill bilateral insertion points at approximately \pm 2 $\mathrm{mm}$ lateral to lambda, and then manually inserting a blunted 25 gauge needle to the base of the brain at a $45^{\circ}$ lateral angle, and then gently tracing the inner wall of the skull back to the insertion point. Of the nine subjects with parahippocampal lesions, three were excluded from analysis because of poor EMG records $(n=2)$ and ventral hippocampal damage $(n=1)$.

\section{Histology}

When the experiments were completed, subjects were overdosed with an intraperitoneal injection of sodium pentobarbital and transcardially perfused with PBS, followed by a $3 \%$ formalin solution. Brains were postfixed for at least $48 \mathrm{~h}$ in a formalin-sucrose solution before being sliced in the sagittal plane (50 $\mu \mathrm{m}$ sections), mounted, and stained with cresyl violet. Light microscopy was then used to identify electrode tracks and to assess the extents of parahippocampal lesions. 


\section{Results}

Intact subjects

To study the temporal relationships between neocortical and hippocampal activity patterns in neonatal rats, we placed $\mathrm{Ag} /$ $\mathrm{AgCl}$ electrodes in the $\mathrm{S} 1$-forelimb and S1-barrel regions of the neocortex, and silicon probes in the CA1 field of the dorsal hippocampus of seven P5-P6 subjects. Five of these subjects provided simultaneous neocortical EEG records from the S1-barrel and S1-forelimb regions; the remaining two subjects provided data only from the S1-forelimb region. Hippocampal unit and LFP activity were recorded from each subject, yielding a total of 100 neurons (14.3 \pm 3.6 neurons/subject; range, 4-31). EMG electrodes were implanted in the forelimb, hindlimb, and nuchal muscles contralateral to the neural recording sites to allow identification of behavioral states and to detect the occurrence of myoclonic twitches. Of the seven subjects studied, five provided simultaneous EMG records from the forelimb, hindlimb, and nuchal muscles; in the remaining two subjects, only two of the three EMG electrodes provided usable data.

Myoclonic twitches in separate muscle groups occur in correlated bouts

As reported previously, the predominant behavioral state expressed at the ages studied here was AS, a state that is characterized by myoclonic twitching against a background of muscle atonia (Gramsbergen et al., 1970; Jouvet-Mounier et al., 1970; Karlsson and Blumberg, 2002). Accordingly, prominent muscle twitches were observed in all three muscle groups examined (Fig. $1 A$ ). To determine the temporal relationships among individual twitches occurring in the three muscle groups, twitch cross-correlograms were constructed for all pairs of simultaneously recorded muscle groups. A total of 17 cross-correlograms were constructed from the available EMG data, and all of them indicated significant $(p<$ $0.0015)$ pairwise correlations among the three muscle groups (Fig. $1 B$ ).

\section{Myoclonic twitches precede interregionally correlated neocortical spindle bursts in $S 1$}

As described previously (Khazipov et al., 2004; Marcano-Reik and Blumberg, 2008), neocortical spindle bursts were robustly expressed during periods of AS-related twitching. For each subject, we used established methods (Mohns and Blumberg, 2008) (see Materials and Methods) to quantify the relationships among spindle bursts in the S1-forelimb and S1-barrel regions and twitching in the three muscle groups sampled. In each subject, spindle burst power in both neocortical regions increased significantly in the period surrounding a twitch in all three muscle groups, consistently reaching peak power in the period immediately after the twitch (Fig. $2 \mathrm{~B}$ ). The mean latency to peak spindle burst power in the $\mathrm{S} 1$-forelimb region was $72.3 \pm 7.7 \mathrm{~ms}$ after forelimb twitches, $40.7 \pm 5.1 \mathrm{~ms}$ after hindlimb twitches, and
$65.7 \pm 11.1 \mathrm{~ms}$ after nuchal twitches. The mean latency to peak spindle burst power in the S1-barrel region was $84.2 \pm 13.5 \mathrm{~ms}$ after forelimb twitches, $61.0 \pm 11.4 \mathrm{~ms}$ after hindlimb twitches, and $56.2 \pm 14.9 \mathrm{~ms}$ after nuchal twitches. The three twitchtriggered spindle burst latencies were not significantly different from one another in either the $S 1$-forelimb region $\left(F_{(2,36)}=1.9\right)$ or the S1-barrel region $\left(F_{(2,31)}=2.7\right)$; in addition, the latencies did not differ significantly between the two neocortical areas $\left(F_{(1,67)}=0.5\right)$. Finally, the magnitude of spindle burst power did not differ significantly after twitches in any of the three muscle groups in either the S1-forelimb region $\left(F_{(2,36)}=2.4\right.$, NS) or the $\mathrm{S} 1$-barrel region $\left(F_{(2,31)}=1.0, \mathrm{NS}\right)$. Figure $2 C$ presents representative data illustrating the tendency for cooccurrence of twitches in the three muscle groups, as well as the tendency for cooccurrence of spindle burst power in the S1-forelimb and S1-barrel regions.

\section{Myoclonic twitches precede correlated bursts of hippocampal unit activity}

Consistent with previous findings (Mohns and Blumberg, 2008), the majority of hippocampal unit activity at the ages studied here was associated with AS (Fig. 3A), with a subset of AS-related units exhibiting a distinct increase in firing rate after the occurrence of a twitch (Fig. 3B). We constructed twitch-triggered event corre- 
A

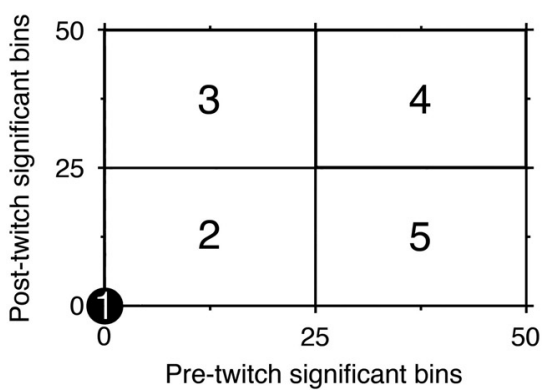

C

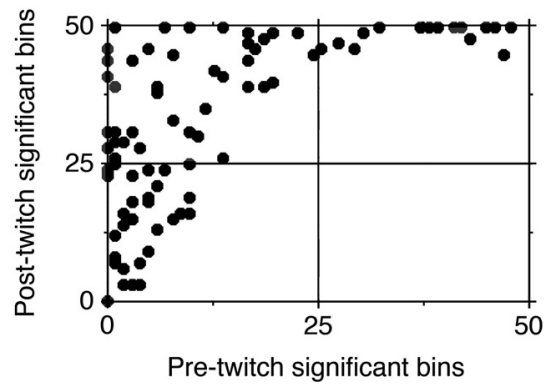

B

\section{Twitch-indifferent}

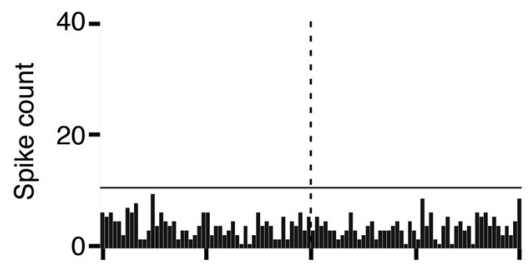

3. Twitch-following

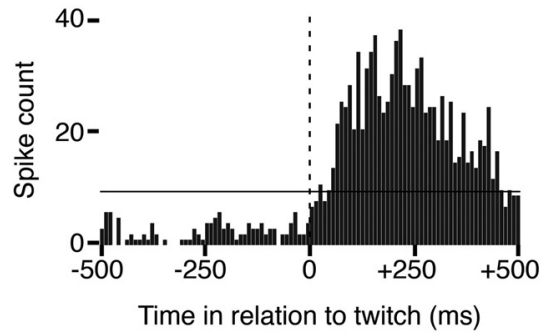

Hindlimb twitches

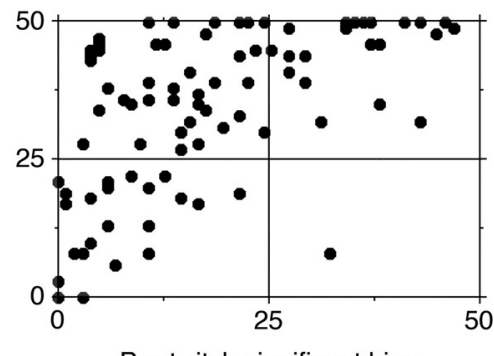

2. Moderately twitch-active

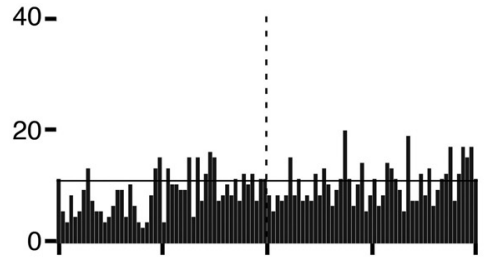

\section{Strongly twitch-active}

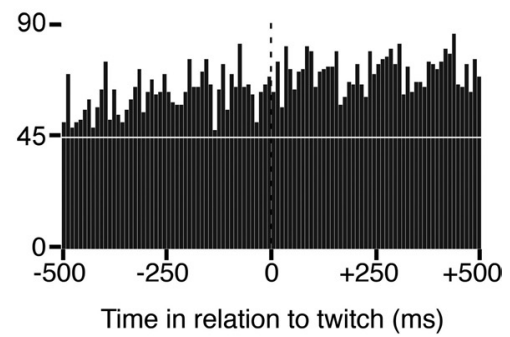

Nuchal twitches

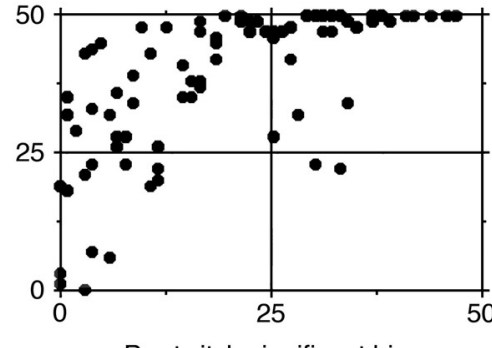

Pre-twitch significant bins

Figure 4. Firing patterns of hippocampal units in relation to twitches. $A$, Diagram illustrating the classification procedure for twitch-related hippocampal unit activity. For each unit, the number of significant pre-twitch bins was plotted against the number of significant post-twitch bins, and units were then defined as belonging to one of five categories. Examples of units within the areas defined in $\boldsymbol{A}$ are shown in $\boldsymbol{B}$. No example is given for area 5 , because twitch-preceding neurons were rarely observed. The vertical dotted lines at 0 ms indicate the time of twitch occurrence; the horizontal lines indicate the expected value +3 SDs $(p<0.0015)$. C, Neuronal firing patterns before and after twitches in the forelimb, hindlimb, and nuchal muscles. For each neuron, the number of pre-twitch significant bins is plotted against the number of post-twitch significant bins; note that, whether neuronal activity is triggered by forelimb, hindlimb, or nuchal twitches, there is a similar distribution of firing profiles.

lations in which unit activity was summed for the period within $\pm 500 \mathrm{~ms}$ ( $10 \mathrm{~ms}$ bins) of a twitch. Based on this analysis, five classes of unit activity were defined, as illustrated in Figure $4 A$ : twitch-indifferent units had no significant bins during the pretwitch or post-twitch period (Fig. $4 A$, area 1); moderately twitchactive units had $>0$ but $<25$ pre-twitch or post-twitch significant bins (area 2); twitch-following units had $\leq 25$ pre-twitch significant bins and $\geq 25$ post-twitch significant bins (area 3 ); strongly twitch-active units had $\geq 25$ pre-twitch significant bins and $>25$ post-twitch significant bins (area 4); and twitch-preceding units had $\geq 25$ pre-twitch significant bins and $<25$ post-twitch significant bins (area 5).

Representative examples of hippocampal unit activity patterns are shown in Figure $4 B$, and the distribution of twitchtriggered units among the five classes of unit activity is shown in Figure 4C. Regardless of muscle group, hippocampal units were predominantly classified as moderately twitch-active, twitchfollowing, or strongly twitch-active; units were rarely classified as twitch-indifferent or twitch-preceding (Table 1). The activity of hippocampal units was significantly intercorrelated (values of $p<0.0001)$ regardless of whether the activity was triggered by twitches of the nuchal, forelimb, or hindlimb muscles, likely reflecting the highly correlated structure of the twitches themselves.

Neocortical spindle bursts precede hippocampal unit activity

Having established that both neocortical spindle bursts and hippocampal unit activity consistently follow twitches, we next investigated whether hippocampal unit activity consistently follows spindle bursts. We found that 98 of the 100 hippocampal units analyzed $(98.0 \%)$ were associated with significant increases in neocortical spindle burst power (Fig. 5A), and in 91 of these 98 units $(92.9 \%)$, spindle burst power 
Table 1. Number of hippocampal units within each activity class for intact and parahippocampal lesioned infant rats

\begin{tabular}{|c|c|c|c|c|c|}
\hline & Twitch-indifferent units & Moderately twitch-active units & Twitch-following units & Strongly twitch-active units & Twitch-preceding units \\
\hline \multicolumn{6}{|l|}{ Intact } \\
\hline Triggered by forelimb twitches ( 89 units) & 1 & 26 & 44 & 18 & 0 \\
\hline Triggered by hindlimb twitches (96 units) & 1 & 22 & 43 & 29 & 1 \\
\hline Triggered by nuchal twitches (100 units) & 0 & 13 & 37 & 48 & 2 \\
\hline \multicolumn{6}{|l|}{ Lesioned } \\
\hline Triggered by forelimb twitches (37 units) & 7 & 29 & 1 & 0 & 0 \\
\hline Triggered by hindlimb twitches ( 24 units) & 9 & 15 & 0 & 0 & 0 \\
\hline Triggered by nuchal twitches (42 units) & 12 & 28 & 2 & 0 & 0 \\
\hline
\end{tabular}

The table displays the number of units in each category when triggered by twitches in the forelimb, hindlimb, and nuchal muscles. Note that, after parahippocampal lesions, the majority of units are either twitch-indifferent or twitch-active.

reached its peak in the period immediately preceding hippocampal unit activity (Fig. 5B). For the 98 units with significant unit-triggered spindle burst power, peak power in the S1-forelimb region was reached $153.4 \pm 10.2 \mathrm{~ms}$ before unit activity, whereas peak power in the S1-barrel region was reached $144.3 \pm 11.5 \mathrm{~ms}$ before unit activity; the difference in latencies between the two neocortical areas was not significant $\left(F_{(1,224)}=2.5, \mathrm{NS}\right)$. There was also no significant difference in the magnitude (in SDs) of unit-triggered spindle burst power between the two neocortical areas $\left(F_{(1,263)}=0.3\right.$, NS).

To investigate whether there were subpopulations of hippocampal units that were preferentially active after increases in either S1-forelimb or S1-barrel spindle burst power, we plotted the unit-triggered magnitude of spindle burst significance in the $\mathrm{S} 1$-forelimb region against that of the S1-barrel region. This analysis revealed a significant linear relationship (Fig. 5C) $\left(r^{2}=0.55 ; p<0.0001\right)$.

We next investigated whether greater spindle burst power was correlated with greater hippocampal unit activity. The number of significant post-twitch hippocampal unit bins was plotted against unit-triggered spindle burst power for both the S1-forelimb and S1-barrel regions. In each case, neocortical and hippocampal activity were positively correlated $(p<0.0001)$, indicating that greater spindle burst power in the neocortex is associated with more unit activity in the hippocampus.

Cooccurrence of neocortical spindle bursts and hippocampal gamma oscillations

As reported previously (Lahtinen et al., 2002; Mohns and Blumberg, 2008), the AS-related hippocampal gamma rhythm emerges gradually during the first postnatal week. Because hippocampal gamma oscillations and neocortical spindle bursts are both present at the ages examined here, we investigated whether bouts of twitching provide an opportunity for early coordination of hippocampal and neocortical EEG rhythms. As illustrated in Figure 6, although AS-related gamma activity was expressed intermittently at these ages (Mohns and Blum-

B versa.
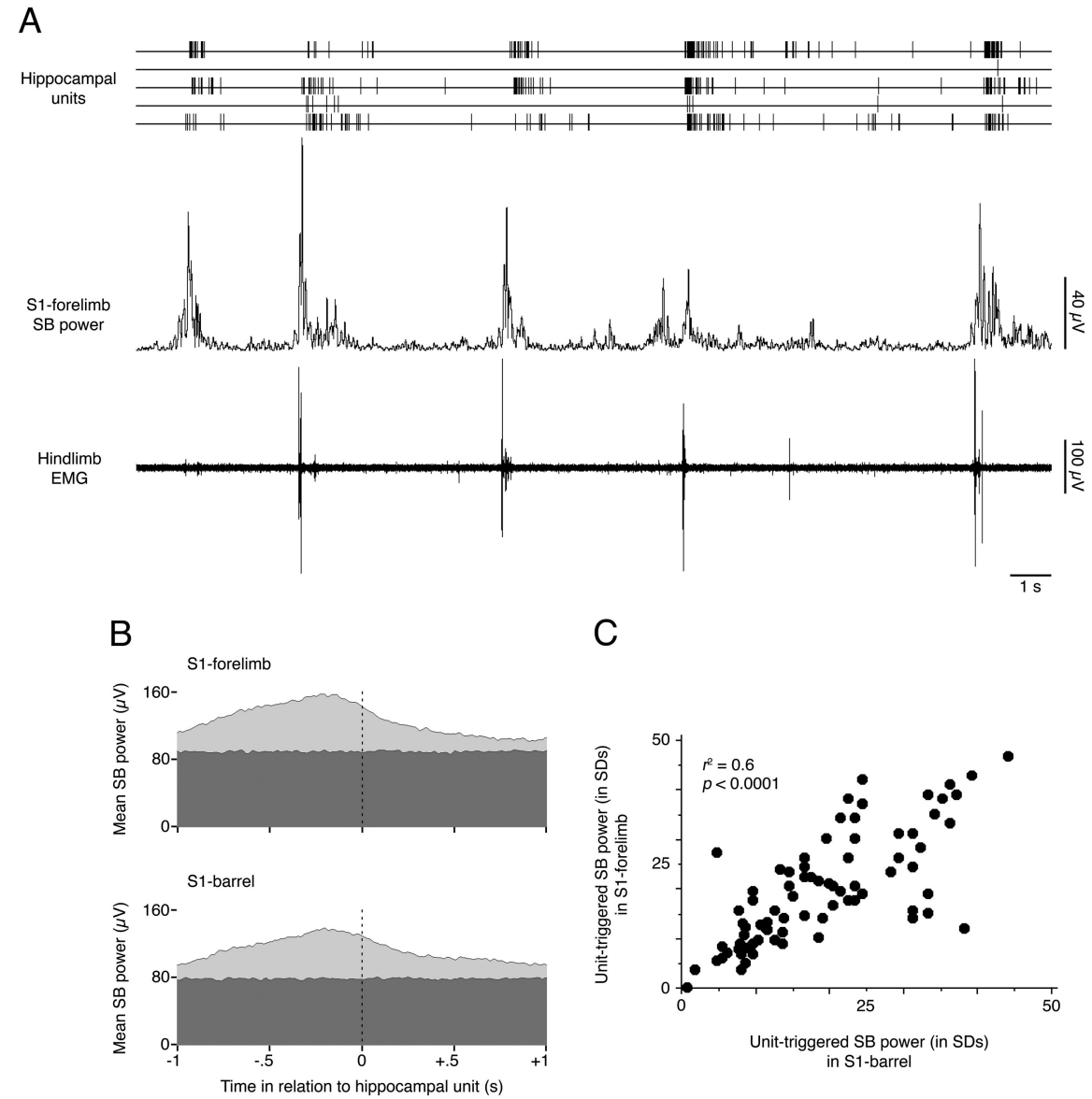

C

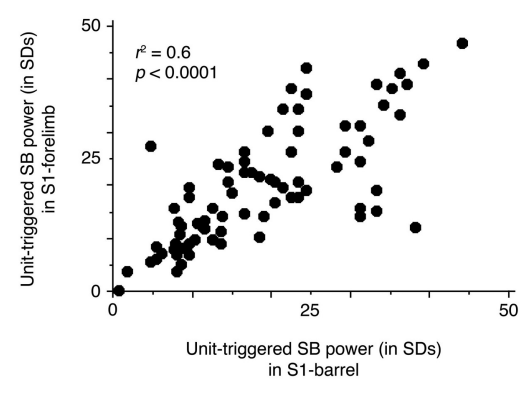

Figure 5. Neocortical spindle bursts consistently precede hippocampal unit activity. $\boldsymbol{A}$, The sequential relationship between muscle twitches, spindle burst power, and hippocampal unit activity is shown. Note that bursts of hippocampal unit activity consistently follow increases in neocortical spindle burst activity and that peak spindle burst activity consistently follows muscle twitches. The tendency for peak spindle burst power to precede hippocampal unit activity is illustrated in $\boldsymbol{B}$; for one hippocampal neuron, mean unit-triggered spindle burst power (light gray) in both the S1-forelimb and S1-barrel regions can be seen reaching its peak before the unit fires (dotted line). Random-triggered spindle burst power is shown in dark gray. C, The magnitudes of hippocampal unit-triggered spindle burst power in the S1-forelimb and S1-barrel regions were positively correlated, indicating that hippocampal neurons were not activated selectively by S1-forelimb spindle bursts rather than S1-barrel spindle bursts, or vice

berg, 2008), when gamma did occur it tended to be coincident with neocortical spindle burst activity.

\section{Responses of the developing hippocampus to exogenous} sensory stimulation

Consistent with previous observations in infant rats (Khazipov et al., 2004; Minlebaev et al., 2007; Marcano-Reik and Blumberg, 2008), all subjects in the present study exhibited increases in S1forelimb and S1-barrel spindle burst power on application of a 


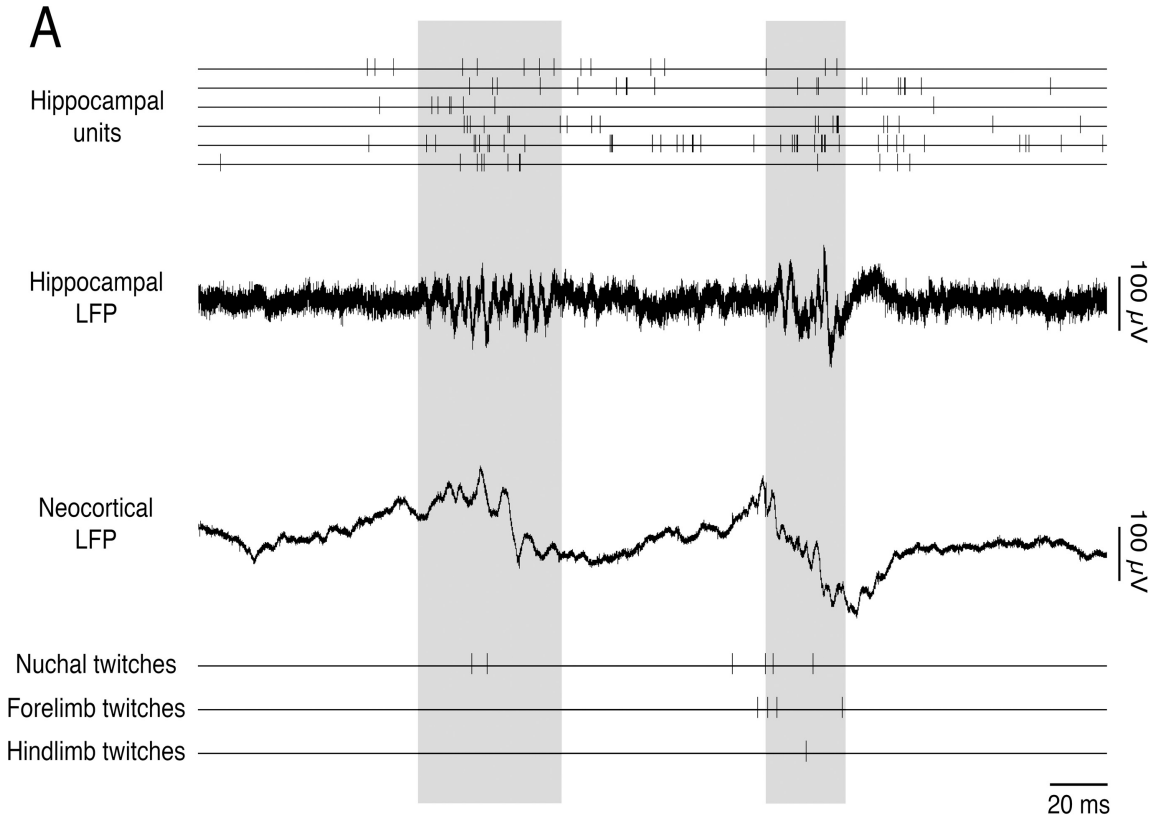

B

Hippocampal units

Neocortical
SB power

Hippocampal gamma powe

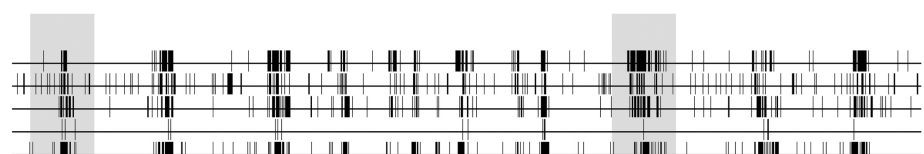

Figure 6. Cooccurrence of neocortical spindle bursts and hippocampal gamma oscillations during active sleep. $\boldsymbol{A}$, Representative record from a $\mathrm{P} 6$ rat showing the relationship among hippocampal unit activity, hippocampal gamma oscillations, neocortical spindle bursts, and muscle twitches. Two periods of coincident hippocampal gamma and neocortical spindle bursts are highlighted in gray. $\boldsymbol{B}$, Representative record from the same P6 rat showing hippocampal unit activity, neocortical spindle burst power, and hippocampal gamma power $(30-100 \mathrm{~Hz})$ at a larger timescale. Note the regular cooccurrence of hippocampal unit activity with neocortical spindle bursts. Although gamma power is weak at this age, when it does occur it tends to be associated with hippocampal unit activity as well as increased spindle burst power. Two prominent examples are highlighted in gray.

sensory stimulus. To determine whether hippocampal units are also responsive to sensory stimulation, 81 of the 100 recorded units were analyzed for their responsiveness to whisker pad stimulation (see Materials and Methods) (19 units were excluded from analysis because of artifact). Of those 81 units, $59(72.8 \%)$ exceeded the criterion for significant responding. Figure 7 illustrates the sequential increase in neocortical spindle burst power and hippocampal unit activity after application of the stimulus.

We next investigated whether the units that were more responsive to evoked stimulation of the whisker pad were also more responsive to spontaneous twitches. Even when significant, these correlations were of only modest magnitude (forelimb: $r^{2}=$ 0.037, NS; hindlimb: $r^{2}=0.078, p=0.014$; nuchal: $r^{2}=0.064$, $p=0.023)$. It therefore appears that twitch responsiveness is only moderately correlated with sensory responsiveness in CA1 neurons. However, it is important to stress that only sensory stimu- lation of the whisker pad was used here; a comparison of neuronal responses to twitches in a limb and sensory stimulation of the same limb may have revealed a stronger correlation.

\section{Effects of parahippocampal lesions on neocortical-to-hippocampal communication}

To determine whether the relationships between developing neocortical and hippocampal activity described above were causal, six subjects were prepared as above, but with the addition of bilateral transections of the parahippocampal region (i.e., entorhinal cortex, subiculum, presubiculum, and parasubiculum), which serves as the primary conduit for activity traveling between the neocortex and hippocampus (Deadwyler et al., 1981; Foster et al., 1988; Burwell, 2000; Lavenex and Amaral, 2000; Witter et al., 2000). The locations of the lesions are illustrated in Figure $8 \mathrm{~A}$. To access the parahippocampal region, all lesions necessarily damaged portions of visual cortex, retrosplenial agranular cortex, and the forceps major of the corpus callosum. Of the six subjects, four provided simultaneous EMG records from the forelimb, hindlimb, and nuchal muscles; in the remaining two subjects, only one or two of the EMG electrodes provided usable data. Each of the subjects provided simultaneous neocortical EEG records from the S1-forelimb and S1barrel regions.

\section{The relationship between spontaneous} muscle twitches and neocortical spindle bursts is unaffected by parahippocampal lesions

As expected, parahippocampal lesions did not affect the correlations among twitches occurring in separate muscle groups. To confirm that the lesions also did not affect the expression of twitch-related neocortical spindle burst activity, we quantified the relationship between spindle bursts in the S1-barrel and S1-forelimb regions and twitching in the three muscle groups. As was the case for intact subjects, spindle burst power in each of the lesioned subjects increased significantly in the period surrounding a twitch, consistently reaching peak power in the period immediately after a twitch. Neither the mean latency to peak spindle burst power nor the magnitude of spindle burst power was affected by the lesions.

\section{Parahippocampal lesions decrease twitch-related hippocampal} unit activity

The activity of 41 hippocampal units ( $6.8 \pm 1.5$ units per subject; range, 4-13) was analyzed from the six lesioned subjects. We again constructed twitch-triggered event correlations in which unit activity was summed for the period within $\pm 500 \mathrm{~ms}$ (in 10 ms bins) of a twitch. Plots were again constructed for each unit in relation to twitches in the forelimb, hindlimb, and nuchal mus- 
cles (Fig. $8 B$ ). Note that, in the lesioned subjects, there were no strongly twitchactive units and very few twitch-following units; indeed, the vast majority of units were now in the moderately twitch-active and twitch-indifferent categories. The percentage of units found in each category for intact and lesioned subjects is illustrated in Figure $9 A$; note that, after parahippocampal lesions, $\sim 70 \%$ of unit activity is moderately twitch-active (Table 1). Accordingly, in subjects with parahippocampal lesions, twitch-triggered unit activity was significantly lower during the $500 \mathrm{~ms}$ after a twitch (intact, $36.4 \pm 0.9$ significant bins; lesioned, $12.9 \pm 1.4$ significant bins; $\left.F_{(1,422)}=220.0, p<0.0001\right)$.

The relationship between neocortical and hippocampal activity is disrupted by parahippocampal lesions

Only 22 of the 41 hippocampal units analyzed $(53.7 \%)$ were now associated with significantly increased spindle burst power, which is significantly lower than observed in intact subjects $\left(F_{(1,224)}=63.5\right.$; $p<0.0001$ ) (Fig. 9B). Furthermore, in only 15 of these 22 units $(68.2 \% ; 36.6 \%$ overall) was peak spindle burst power still reached in the $500 \mathrm{~ms}$ period preceding hippocampal unit activity. Thus, the consistent sequential occurrence of neocortical spindle bursts and hippocampal unit activity observed in intact subjects was significantly disrupted by parahippocampal lesions.

Evoked responses of the neocortex and hippocampus after

parahippocampal lesions

To determine whether damage to the parahippocampal region lessened neocortical and/or hippocampal responses to exogenous sensory stimulation, brush strokes were again applied to the whisker pad. In all five lesioned subjects tested in this way, the stimulus was followed by a significant $(p<$ $0.0015)$ increase in spindle burst power in the S1-forelimb region as well as in the S1barrel region. The mean magnitudes of spindle burst power after stimulus application (S1-forelimb, $10.4 \pm$ 3.0 SDs; S1-barrel, $14.2 \pm 7.2$ SDs) did not differ significantly between the two neocortical regions $\left(F_{(1,18)}=0.2\right.$, NS), nor did they differ significantly from the responses observed in intact subjects $\left(F_{(1,18)}=0.1, \mathrm{NS}\right)$. Thus, parahippocampal lesions did not significantly affect the neocortical response to exogenous sensory stimulation.

Of the 41 hippocampal units analyzed in these five lesioned subjects, 24 were examined for their responses to sensory stimulation. Of these 24 units, only 8 (33.3\%) were significantly responsive. Moreover, the mean number of significant bins observed after stimulus presentation was significantly lower in the lesioned group than in the intact group (intact, $12.0 \pm 1.2$; lesion, $\left.4.1 \pm 1.4 ; F_{(1,219)}=30.3, p<0.0001\right)$. Representative data from a typical lesioned subject are presented in Figure 9C; note that, although the neocortical spindle burst response to the stimulus is still prominently expressed, the hippocampal unit activity is now sparse and not consistently related to the stimulus.

\section{Discussion}

It has been hypothesized that AS-related myoclonic twitches play a functional role in the establishment of somatotopy and sensory representation within the spinal cord (Petersson et al., 2003) and neocortex (Khazipov et al., 2004). Twitches of the distal limbs generate tactile and proprioceptive feedback that, at least in part, trigger spindle bursts in neocortex (Khazipov et al., 2004; Marcano-Reik and Blumberg, 2008). Furthermore, as we recently reported (Mohns and Blumberg, 2008), the activity of the developing hippocampus also exhibits a 
A Intact subjects

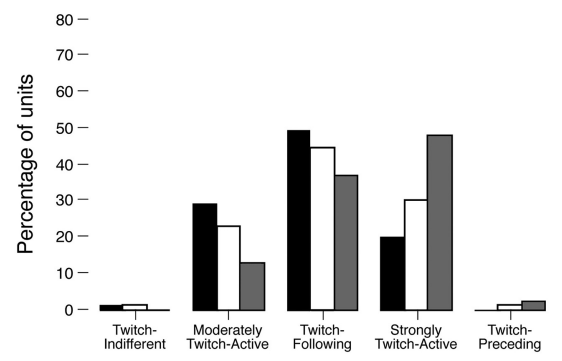

B
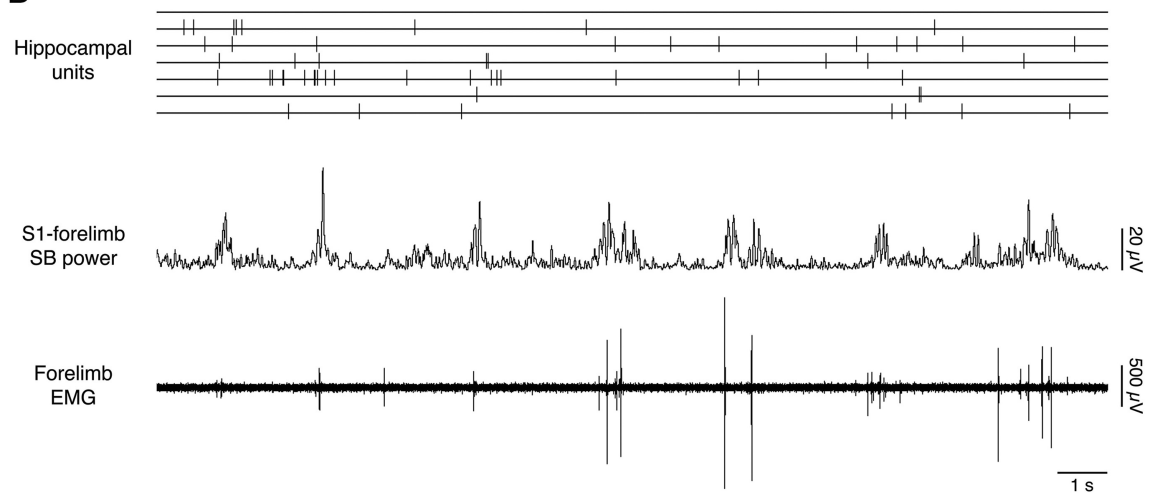

C Hippocampa units

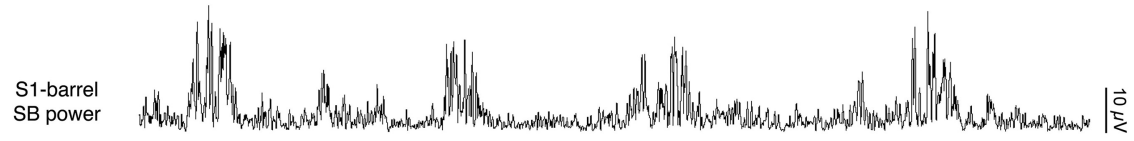

Whisker pad stimulations

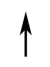

$\uparrow$

$\uparrow$

Figure 9. Effects of parahippocampal lesions on hippocampal and neocortical activity. $\boldsymbol{A}$, Bar graphs show the percentages of units found in each activity category for intact (left) and lesioned (right) subjects. Data are presented for unit activity triggered by forelimb, hindlimb, and nuchal twitches. Note that, in the lesioned subjects, there are no strongly twitch-active units and very few twitch-following units; indeed, the vast majority of units are now found in the moderately twitch-active and twitch-indifferent categories. $\boldsymbol{B}$, Representative record of hippocampal unit activity, neocortical spindle burst power, and forelimb EMG recorded from a subject with parahippocampal lesions. Whereas the lesion does not disrupt the association between twitching and neocortical spindle burst power, the association with hippocampal unit activity is severely weakened. C, Representative record of hippocampal and neocortical responses to whisker pad stimulation after parahippocampal lesions. Whisker pad stimulations (arrows) continue to cause significant increases in neocortical spindle burst power; in contrast, hippocampal unit activity is no longer consistently evoked.

close temporal association with twitching. Based on these findings, we hypothesized that the sensory feedback arising from twitches triggers neocortical spindle bursts that in turn activate the hippocampus, thereby providing an early form of neocortical-hippocampal communication.

The present results provide direct support for that hypothesis. Specifically, we found that the predominantly AS-related neuronal activity of the hippocampus consistently increased after neocortical spindle bursts and that greater spindle burst power was positively correlated with greater hippocampal unit activity. Application of exogenous sensory stimulation produced the same sequence of neocortical-to-hippocampal activation, demonstrating for the first time that the neonatal hippocampus is responsive to sensory input. Surgical separation of the neocortex from the hippocampus did not significantly affect the expression of neocortical spindle bursts but did disrupt $\uparrow$

the temporal association between neocortical spindle bursts and hippocampal activity. These lesions also disrupted the hippocampal response to sensory stimulation.

AS-related twitches occurring in the ipsilateral forelimb, hindlimb, and nuchal muscles were significantly correlated with one another within a window of $\pm 100 \mathrm{~ms}$. This result is consistent with the findings of Robinson et al. (2000), who reported that, after a visually observed twitch in one limb, there was a high probability of another twitch occurring in another limb within 100-500 ms. In the present study, it appears that this highly correlated twitching activity resulted in spindle bursts that were produced nearly simultaneously in the forelimb and barrel regions of S1. Thus, the correlated structure of twitching may have precluded our detection of somatotopically selective feedback responses in neocortex. In developing rats, sensory feedback from correlated twitches in multiple muscle groups may help to facilitate the initial formation of functional neocortical circuits among sensory regions.

Neocortical activity has been shown to precede hippocampal activity in adult rats by $30-100 \mathrm{~ms}$ during QS (Sirota et al., 2003; Mölle et al., 2006; Ji and Wilson, 2007); conversely, hippocampal activity can also precede neocortical activity during QS, AS, and active wakefulness (Siapas and Wilson, 1998; Siapas et al., 2005; Sirota et al., 2008). In neonates, we found that the activity of the neocortex precedes that of the hippocampus by $144-153 \mathrm{~ms}$ during AS; however, hippocampal activation of the neocortex was not observed here during AS. The latter type of activation may emerge later in development or may occur in neocortical and/or hippocampal regions that were not recorded from in the present study.

Because spindle bursts are associated with bursts of neocortical unit activity (Khazipov et al., 2004; Yang et al., 2009), the observation that they consistently precede bursts of hippocampal unit activity may have important implications for our understanding of activity-dependent development and refinement of the circuits linking these two regions. Such correlated activity may provide an opportunity for "Hebbian" synaptic plasticity, which has been implicated in many learning and developmental processes (Buonomano and Merzenich, 1998; Bi and Poo, 2001). The sequential, as well as overlapping, correlated bursts of activity may provide the necessary temporal window in which both strengthening and removal of neonatal circuitry can take place.

Parahippocampal lesions disrupted the temporal relationship between neocortical spindle bursts and hippocampal unit activity, thereby supporting the hypothesis that the neocortex activates the hippocampus at these ages. This finding is consistent 
with available anatomical data, which indicate that entorhinalto-hippocampal projections are present even prenatally (Supèr and Soriano, 1994; Deng et al., 2006). In lesioned subjects, there was some residual temporal association between neocortical and hippocampal activity, perhaps attributable to incomplete parahippocampal lesions. Alternatively, it is possible that this residual association resulted from independent sleep-related modulation of neocortical and hippocampal activity by the basal forebrain. Indeed, in adult rats, sensory information is thought to be conveyed to the hippocampus via parahippocampal inputs (Burwell, 2000; Witter et al., 2000), whereas the basal forebrain is thought to provide state-dependent modulation of both hippocampal activity (Bland and Oddie, 2001; Buzsáki, 2002; Vertes et al., 2004) and neocortical activity (Sterman and Clemente, 1962; Szymusiak and McGinty, 1989; Lee et al., 2004). Thus, the twitch-following and strongly twitch-active hippocampal units described here may represent populations of sensory-responsive cells that are activated via the parahippocampal region, whereas the moderately twitch-active neurons may represent a population of statedependent cells that is activated via the basal forebrain.

Stimulation of the whisker pad elicited bursts of unit activity in the hippocampus, indicating that the developing hippocampus is responsive to sensory input, just as is the adult hippocampus (Wood et al., 1999; Vinogradova, 2001; Pereira et al., 2007). That a large portion of this sensory activation in the neonate appears to be related to twitching may indicate that spontaneous motor activity plays a key role in the emergence of hippocampal sensorimotor integration (Bland and Oddie, 2001). Moreover, in light of hypotheses linking sensory feedback from twitch movements to the development and refinement of neocortical circuits (Khazipov et al., 2004), the demonstration here that sensory feedback from sleep-related twitches sequentially activates the neocortex and hippocampus suggests a functional role for twitching not only in the activity-dependent development of the two structures but also in the development of those cooperative interactions that must develop to support the complex cognitive tasks subserved by these forebrain structures (Squire, 1992; ZolaMorgan and Squire, 1993; Aggleton et al., 2000; Lavenex and Amaral, 2000; Rolls, 2000). Finally, the current findings may inform efforts aimed at understanding those early neocortical-hippocampal processes that make possible the development of egocentric maps and, in turn, the emergence of effective spatial navigation (McNaughton et al., 2006; Moser et al., 2008).

\section{References}

Aggleton JP, Vann SD, Oswald CJ, Good M (2000) Identifying cortical inputs to the rat hippocampus that subserve allocentric spatial processes: a simple problem with a complex answer. Hippocampus 10:466-474.

Bi G, Poo M (2001) Synaptic modification by correlated activity: Hebb's postulate revisited. Annu Rev Neurosci 24:139-166.

Bland BH, Oddie SD (2001) Theta band oscillation and synchrony in the hippocampal formation and associated structures: the case for its role in sensorimotor integration. Behav Brain Res 127:119-136.

Buonomano DV, Merzenich MM (1998) Cortical plasticity: from synapses to maps. Annu Rev Neurosci 21:149-186.

Burwell RD (2000) The parahippocampal region: corticocortical connectivity. Ann N Y Acad Sci 911:25-42.

Buzsáki G (2002) Theta oscillations in the hippocampus. Neuron 33:325-340.

Corner MA, Kwee P (1976) Cyclic EEG and motility patterns during sleep in restrained infant rats. Electroencephalogr Clin Neurophysiol 41:64-72.

Corner MA, van Pelt J, Wolters PS, Baker RE, Nuytinck RH (2002) Physiological effects of sustained blockade of excitatory synaptic transmission on spontaneously active developing neuronal networks - an inquiry into the reciprocal linkage between intrinsic biorhythms and neuroplasticity in early ontogeny. Neurosci Biobehav Rev 26:127-185.
Deadwyler SA, West MO, Robinson JH (1981) Entorhinal and septal inputs differentially control sensory-evoked responses in the rat dentate gyrus. Science 211:1181-1183.

Deng JB, Yu DM, Li MS (2006) Formation of the entorhino-hippocampal pathway: a tracing study in vitro and in vivo. Neurosci Bull 22:305-314.

Eggermont JJ (1992) Neural interaction in cat primary auditory cortex. Dependence on recording depth, electrode separation, and age. J Neurophysiololgy 68:1216-1228.

Feller MB (1999) Spontaneous correlated activity in developing neural circuits. Neuron 22:653-656.

Foster TC, Hampson RE, West MO, Deadwyler SA (1988) Control of sensory activation of granule cells in the fascia dentata by extrinsic afferents: septal and entorhinal inputs. J Neurosci 8:3869-3878.

Gramsbergen A, Schwartze P, Prechtl HF (1970) The postnatal development of behavioral states in the rat. Dev Psychobiol 3:267-280.

Hahn TT, Sakmann B, Mehta MR (2006) Phase-locking of hippocampal interneurons' membrane potential to neocortical up-down states. Nat Neurosci 9:1359-1361.

Hahn TT, Sakmann B, Mehta MR (2007) Differential responses of hippocampal subfields to cortical up-down states. Proc Natl Acad Sci U S A 104:5169-5174.

Isomura Y, Sirota A, Ozen S, Montgomery S, Mizuseki K, Henze DA, Buzsáki G (2006) Integration and segregation of activity in entorhinal-hippocampal subregions by neocortical slow oscillations. Neuron 52:871-882.

Ji D, Wilson MA (2007) Coordinated memory replay in the visual cortex and hippocampus during sleep. Nat Neurosci 10:100-107.

Jones MW, Wilson MA (2005) Theta rhythms coordinate hippocampalprefrontal interactions in a spatial memory task. PLoS Biol 3:e402.

Jouvet-Mounier D, Astic L, Lacote D (1970) Ontogenesis of the states of sleep in rat, cat, and guinea pig during the first postnatal month. Dev Psychobiol 2:216-239.

Karlsson Æ, Blumberg MS (2002) The union of the state: myoclonic twitching is coupled with nuchal muscle atonia in infant rats. Behav Neurosci 116:912-917.

Karlsson Æ, Gall AJ, Mohns EJ, Seelke AM, Blumberg MS (2005) The neural substrates of infant sleep in rats. PLoS Biol 3:e143.

Katz LC, Shatz CJ (1996) Synaptic activity and the construction of cortical circuits. Science 274:1133-1138.

Khazipov R, Sirota A, Leinekugel X, Holmes GL, Ben-Ari Y, Buzsáki G (2004) Early motor activity drives spindle bursts in the developing somatosensory cortex. Nature 432:758-761.

Kirov SA, Goddard CA, Harris KM (2004) Age-dependence in the homeostatic upregulation of hippocampal dendritic spine number during blocked synaptic transmission. Neuropharmacology 47:640-648.

Lahtinen H, Palva JM, Sumanen S, Voipio J, Kaila K, Taira T (2002) Postnatal development of rat hippocampal gamma rhythm in vivo. J Neurophysiol 88:1469-1474.

Lauri SE, Lamsa K, Pavlov I, Riekki R, Johnson BE, Molnar E, Rauvala H, Taira T (2003) Activity blockade increases the number of functional synapses in the hippocampus of newborn rats. Mol Cell Neurosci 22:107-117.

Lavenex P, Amaral DG (2000) Hippocampal-neocortical interaction: a hierarchy of associativity. Hippocampus 10:420-430.

Le Bé JV, Markram H (2006) Spontaneous and evoked synaptic rewiring in the neonatal neocortex. Proc Natl Acad Sci U S A 103:13214-13219.

Lee MG, Manns ID, Alonso A, Jones BE (2004) Sleep-wake related discharge properties of basal forebrain neurons recorded with micropipettes in head-fixed rats. J Neurophysiol 92:1182-1198.

Leinekugel X, Khazipov R, Cannon R, Hirase H, Ben-Ari Y, Buzsáki G (2002) Correlated bursts of activity in the neonatal hippocampus in vivo. Science 296:2049-2052.

Marcano-Reik AJ, Blumberg MS (2008) The corpus callosum modulates spindle-burst activity within homotopic regions of somatosensory cortex in newborn rats. Eur J Neurosci 28:1457-1466.

McNaughton BL, Battaglia FP, Jensen O, Moser EI, Moser MB (2006) Path integration and the neural basis of the "cognitive map." Nat Rev Neurosci 7:663-678.

Minlebaev M, Ben-Ari Y, Khazipov R (2007) Network mechanisms of spindle-burst oscillations in the neonatal rat barrel cortex in vivo. J Neurophysiol 97:692-700.

Mohns EJ, Blumberg MS (2008) Synchronous bursts of neuronal activity in 
the developing hippocampus: modulation by active sleep and association with emerging gamma and theta rhythms. J Neurosci 28:10134-10144.

Mölle M, Yeshenko O, Marshall L, Sara SJ, Born J (2006) Hippocampal sharp wave-ripples linked to slow oscillations in rat slow-wave sleep. J Neurophysiol 96:62-70.

Moser EI, Kropff E, Moser MB (2008) Place cells, grid cells, and the brain's spatial representation system. Annu Rev Neurosci 31:69-89.

O'Donovan MJ (1999) The origin of spontaneous activity in developing networks of the vertebrate nervous system. Curr Opin Neurobiol 9:94-104.

Pereira A, Ribeiro S, Wiest M, Moore LC, Pantoja J, Lin SC, Nicolelis MA (2007) Processing of tactile information by the hippocampus. Proc Natl Acad Sci U S A 104:18286-18291.

Petersson P, Waldenström A, Fåhraeus C, Schouenborg J (2003) Spontaneous muscle twitches during sleep guide spinal self-organization. Nature 424:72-75.

Robinson SR, Blumberg MS, Lane MS, Kreber LA (2000) Spontaneous motor activity in fetal and infant rats is organized into discrete multilimb bouts. Behav Neurosci 114:328-336.

Rolls ET (2000) Hippocampo-cortical and cortico-cortical backprojections. Hippocampus 10:380-388.

Seelke AM, Blumberg MS (2008) The microstructure of active and quiet sleep as cortical delta activity emerges in infant rats. Sleep 31:691-699.

Siapas AG, Wilson MA (1998) Coordinated interactions between hippocampal ripples and cortical spindles during slow-wave sleep. Neuron 21:1123-1128.

Siapas AG, Lubenov EV, Wilson MA (2005) Prefrontal phase locking to hippocampal theta oscillations. Neuron 46:141-151.

Sirota A, Csicsvari J, Buhl D, Buzsáki G (2003) Communication between neocortex and hippocampus during sleep in rodents. Proc Natl Acad Sci U S A 100:2065-2069.
Sirota A, Montgomery S, Fujisawa S, Isomura Y, Zugaro M, Buzsáki G (2008) Entrainment of neocortical neurons and gamma oscillations by the hippocampal theta rhythm. Neuron 60:683-697.

Squire LR (1992) Memory and the hippocampus: a synthesis from findings with rats, monkeys, and humans. Psychol Rev 99:195-231.

Sterman MB, Clemente CD (1962) Forebrain inhibitory mechanisms: sleep patterns induced by basal forebrain stimulation in the behaving cat. Exp Neurol 6:103-117.

Supèr H, Soriano E (1994) The organization of the embryonic and early postnatal murine hippocampus. II. Development of entorhinal, commissural, and septal connections studied with the lipophilic tracer DiI J Comp Neurol 344:101-120.

Szymusiak R, McGinty D (1989) Sleep-waking discharge of basal forebrain projection neurons in cats. Brain Res Bull 22:423-430.

Vertes RP, Hoover WB, Viana Di Prisco G (2004) Theta rhythm of the hippocampus: subcortical control and functional significance. Behav Cogn Neurosci Rev 3:173-200.

Vinogradova OS (2001) Hippocampus as comparator: role of the two input and two output systems of the hippocampus in selection and registration of information. Hippocampus 11:578-598.

Witter MP, Wouterlood FG, Naber PA, Van Haeften T (2000) Anatomical organization of the parahippocampal-hippocampal network. Ann N Y Acad Sci 911:1-24.

Wood ER, Dudchenko PA, Eichenbaum H (1999) The global record of memory in hippocampal neuronal activity. Nature 397:613-616.

Yang JW, Hanganu-Opatz IL, Sun JJ, Luhmann HJ (2009) Three patterns of oscillatory activity differentially synchronize developing neocortical networks in vivo. J Neurosci 29:9011-9025.

Zola-Morgan S, Squire LR (1993) Neuroanatomy of memory. Annu Rev Neurosci 16:547-563. 